\title{
Cell proliferation-related genetic polymorphisms and gastric cancer risk: systematic review and meta-analysis
}

\author{
Lei Gao ${ }^{1}$, Alexandra Nieters ${ }^{2}$ and Hermann Brenner ${ }^{*}, 1$ \\ ${ }^{1}$ Division of Clinical Epidemiology and Aging Research, German Cancer Research Center, Heidelberg, Germany; \\ ${ }^{2}$ Division of Cancer Epidemiology, German Cancer Research Center, Heidelberg, Germany
}

\begin{abstract}
Apart from Helicobacter pylori infection and lifestyle factors, host genetic susceptibility has been suggested to contribute to individual variation in gastric cancer risk as well. Aiming to evaluate the associations between host cell proliferation-related genetic polymorphisms and gastric cancer susceptibility, we reviewed the related studies published until 15 September 2008 and quantitatively summarized the associations of the most widely studied polymorphisms (TP53 Arg72Pro, L-myc EcoRI) using meta-analysis. Fifty-five eligible studies were included in this review. Twenty-three polymorphisms significantly related to gastric cancer risk in at least one study were identified. Polymorphisms determining higher levels of growth factors, which are important for tissue repair, were recently observed to be associated with reduced risk of gastric cancer. In the meta-analysis, TP53 72Pro was associated with increased risk of diffuse gastric cancer among Asians (OR, 1.44; 95\% Cl, 1.04-1.99), but decreased risk of intestinal gastric cancer among Caucasians (OR, 0.56; $95 \% \mathrm{Cl}, 0.36-0.89$ ). This review suggests that cell proliferation-related genetic polymorphisms could be candidate biomarkers of gastric cancer risk, but current evidence for the use for risk stratification is still very limited. Modestly significant associations in meta-analyses stratified by population or type of gastric cancer may be observed by chance because of the limited number of studies and small sample size. Larger studies are warranted to clarify the effect of cell proliferation-related genetic polymorphisms on gastric carcinogenesis.
\end{abstract}

European Journal of Human Genetics (2009) 17, 1658-1667; doi:10.1038/ejhg.2009.102; published online 17 June 2009

Keywords: gastric cancer; genetic polymorphism; cell proliferation

\section{Introduction}

Despite a worldwide decline in incidence, gastric cancer (GC) still is the second most common cause of cancerrelated mortality. ${ }^{1}$ It is well accepted that Helicobacter pylori infection is a key risk factor for GC. However, only a small fraction of the infected people develop GC or its

${ }^{*}$ Correspondence: Dr H Brenner, Division of Clinical Epidemiology and Aging Research, German Cancer Research Center, Bergheimer Str. 20, D-69115 Heidelberg, Germany.

Tel: + 496221 548140; Fax: + 496221548142 ;

E-mail: h.brenner@dkfz-heidelberg.de

Received 2 October 2008; revised 20 March 2009; accepted 14 May 2009; published online 17 June 2009 precursors. $^{2-4}$ Such clinical diversity suggests that factors other than bacterial infection alone determine gastric carcinogenesis. Apart from virulence factors of the pathogen and other environmental and lifestyle risk factors, host genetic susceptibility is also likely to contribute. ${ }^{5-7}$ In recent years, host genetic polymorphisms involved in inflammatory response, carcinogen metabolism, antioxidant protection, mucosal protection and cell proliferation regulation have been widely studied as potential biomarkers to predict GC risk. However, the findings are frequently heterogeneous. ${ }^{8-10}$ In this article, we provide a systematic review of studies addressing the association of cell proliferation-related polymorphisms with GC suscepti- 
bility. For the most widely studied polymorphisms, we also quantitatively summarized associations with GC using meta-analysis.

\section{Materials and methods \\ Literature search}

Studies investigating associations of host genetic polymorphisms and GC risk were identified by searching for articles in the MEDLINE database and Chinese BioMedical Literature Database. Articles published until 15 September 2008 were considered. Different combinations of the key words 'gastric cancer', 'stomach cancer', 'polymorphism(s)' and 'susceptibility' were used to screen for potentially relevant studies. Additional studies were also identified using cross-referencing.

\section{Inclusion and exclusion criteria}

Polymorphisms related to GC risk were divided into several groups according to their biological roles: mucosal protection and inflammation response, carcinogen metabolism, oxidative damage and DNA reparation, tumor invasion (cell adhesion and angiogenesis) and the regulation of cell proliferation. Cell proliferation-related genetic polymorphisms were selected for this review. Case-control or cohort studies presenting original data on associations between the genetic polymorphisms and GC were included. If the effect of a polymorphism was reported in duplicate, the article published in English or published earlier was included. Exclusion criteria include: (i) articles not in English or Chinese; (ii) review articles; (iii) articles which were not cases-control or cohort studies addressing GC susceptibility; (iv) articles focusing on polymorphisms other than cell proliferation-related.

\section{Data extraction and statistical analysis}

Data extraction and analysis followed standard methods for systematic review and meta-analysis ${ }^{11,12}$ as described elsewhere. ${ }^{13}$ Briefly, for all studies, we extracted the following data from the original publications: first author and year of publication; genes and relevant polymorphisms; characteristics of the study design and the study population, association according to tumor location and histological types, case-control matching criteria and covariates controlled for (the latter are presented in Supplementary Tables only). Adjusted odds ratios (ORs) were extracted from the studies where available and included in tabular presentation. For some studies, crude ORs had to be calculated from the reported frequencies of genotype by disease status.

Meta-analyses were carried out using Comprehensive Meta-Analysis (V2.0, Biostat, Englewood, NJ, USA) for the most widely studied polymorphisms, which were evaluated in at least five studies. Dominant models were used to represent the effect of the polymorphism, and correspond- ing ORs (adjusted ORs were used where available) from the included studies were summarized. Random effects models were used in meta-analysis, taking into account the possibility of heterogeneity between studies, which was evaluated with the $\mathrm{Q}$ test $(P<0.10$ was considered indicative of statistically significant heterogeneity) and the $I^{2}$ statistic (values of 25, 50 and $75 \%$ are considered to represent low, medium and high heterogeneity, respectively). The Begg rank correlation method and the Egger weighted regression method were used to statistically assess publication bias $(P<0.05$ was considered indicative of statistically significant publication bias). Stratified analyses were conducted according to the ethnicity of the study population (Asian or Caucasian) and histological type of GC (Lauren's classification: diffuse type or intestinal type).

\section{Results}

The literature search identified 61 original articles ${ }^{14-74}$ on associations between cell proliferation-related genetic polymorphisms and GC (flow diagram of study identification shown in Figure 1). Of these, six articles ${ }^{69-74}$ were excluded from this review because of duplication reporting of results. From 55 included studies, 54 were hospital-based $(n=46)$ or population-based $(n=8)$ casecontrol studies, and only one was a cohort study-based nested case-control study. ${ }^{34}$ Forty polymorphisms in 27 genes were assessed in included studies and 23 of them were significantly related to GC in at least one study. On the basis of their biological roles, the involved polymorphisms were categorized in two groups: cell cycle and apoptosis regulators (Table 1 and Supplementary Table 1) and cell growth factors-related (Table 2 and Supplementary Table 2) polymorphisms.

There were 43 studies focusing on the relationships between cell cycle regulators-related polymorphisms and GC susceptibility (Table 1). Inconsistent associations were observed for tumor protein p53 (TP53) Arg73Pro, the most widely studied polymorphism. Meta-analysis of the 14 included studies showed that, overall, the Pro allele at codon 72 of TP53 was not significantly associated with GC (OR, 1.08; 95\% confidence interval (CI), 0.91-1.27) (Figure 2). ${ }^{14-23,25-28}$ However, when stratified by ethnicity and histological type of GC, we found that among Asians, the Pro allele acted as risk factor of GC and this association was particularly pronounced in diffuse GC (OR, 1.44; 95\% CI, 1.04-1.99) but absent in intestinal GC (OR, 1.07; 95\% CI, 0.64-1.80). No substantial difference was observed between hospital-based (OR, 1.26; 95\% CI, 1.03-1.53) and population-based studies (OR, 1.13; 95\% CI, 0.87-1.47) among Asians. Among Caucasians, the Pro allele was associated with a reduced risk of intestinal GC (OR, 0.56; 95\% CI, 0.36-0.89), and no significant association was found with diffuse GC (OR, 0.74; 95\% CI, 0.48-1.16). 
1331 abstracts on polymorphisms and gastric cancer

$\mid \rightarrow \begin{aligned} & 897 \text { were excluded for the following reasons: } \\ & 111 \text { were not in English or Chinese } \\ & 128 \text { were review papers } \\ & 658 \text { were not case-control or cohort studies on gastric cancer }\end{aligned}$

434 original articles were identified

$\rightarrow \begin{aligned} & 373 \text { were excluded for the following reasons: } \\ & 172 \text { on mucosal protection and inflammation genes } \\ & 102 \text { on carcinogen metabolism genes } \\ & 61 \text { on oxditive damage and DNA repairment genes } \\ & 28 \text { on tumor invasion genes }\end{aligned}$

61 articles on cell proliferation-related polymorphisms

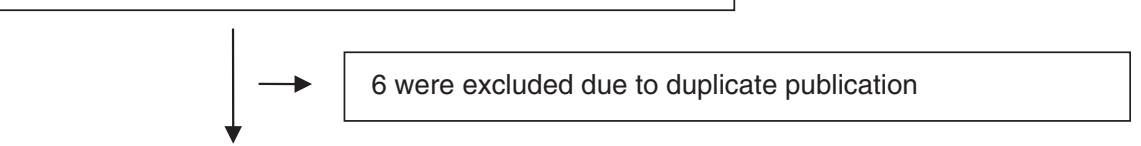

55 studies were included

Figure 1 Flow diagram of study identification (until 15 September 2008).

However, only six out of the 14 included studies reported associations by histological type of GC (four in Asian populations $^{15,20,26,27}$ and two in the Caucasian populations ${ }^{16,25}$ ), and therefore the summarized results have to be interpreted with caution. One study on this polymorphism shown in Table 1 was excluded from the meta-analysis because the genotype frequencies and ORs were not reported by the original article. ${ }^{24}$ No evident publication bias was observed as assessed by Begg rank correlation method $(P=0.83)$ and Egger weighted regression method $(P=0.82)$.

Polymorphisms of functional regulators of TP53, TP53BP2 (tumor protein p53 binding protein 2) and MDM2 were also found to be related to the development of GC in studies from Korea and Japan, respectively. ${ }^{29,30}$ An SNP (exon $24 \mathrm{G}>\mathrm{A}$ ) in TP73, which encodes a homology of p53, showed significant association in Chinese ${ }^{32}$ but not in Japanese. ${ }^{14}$ Four studies were carried out on the $870 \mathrm{G}>\mathrm{A}$ variant in Cyclin $D^{33-36}$ a significantly reduced risk of GC was observed for AA genotype in one study ${ }^{36}$ carried out in the Chinese population. H-RAS was reported to stabilize p21 by promoting the formation of p21-cyclin D1 complexes that prevent subsequent degradation; Harvey retrovirus-associated DNA sequences (H-RAS) 81C allele was associated with an increased risk of GC (OR, 3.7; 95\% CI, 2.2-6.0) in a study from China. ${ }^{38}$

Meta-analysis showed inconsistent results for the $S$ allele of an EcoRI polymorphism in the second intron of L-myc (MYCL1) (Figure 3). Of the four included Asian studies, one showed a significant increase in risk, the other three presented non-significant associations in opposite directions. ${ }^{39,40,42,45}$ Diverse results were also found in the two studies involving Caucasians. ${ }^{43,44}$ One study on this polymorphism shown in Table 1 was excluded from meta-analysis because the allele frequencies were not reported by the original article. ${ }^{41}$ The results of Begg rank correlation analysis $(P=0.13)$ and Egger weighted regression analysis $(P=0.49)$ did not indicate significant publication bias.

Genotypes inhibiting apoptosis were reported to increase GC risk. For example, Survivin -31C allele was associated with an increased risk compared with GG genotype in studies from China. ${ }^{46,47}$ Gene variants in transcription factors participating in cell cycle regulation, such as peroxisome proliferator-activated receptor $\gamma$ (PPAR- $\gamma$ ), eukaryotic translation release factor 3 , krüppel-like factor 6 and RUNX3, were also identified as potential predictors of GC. ${ }^{49-54}$ 
Table 1 Association between cell cycle and apoptosis regulators gene polymorphisms and gastric cancer susceptibility

\begin{tabular}{|c|c|c|c|c|c|c|}
\hline \multirow[b]{2}{*}{ Polymorphism (SNP ID) } & \multirow[b]{2}{*}{ First author, publish year } & & study design a & tion & Association & GC \\
\hline & & No. of cases/controls & Population & Setting & Groups compared & OR $(95 \% \mathrm{Cl})$ \\
\hline (a) TP53 and its functional regulators & & & & & & \\
\hline TP53 Arg72Pro (rs1042522) & Hamajima, 2002 & $144 / 241$ & Japanese & Hospital & *Pro vs Arg/Arg & $1.1(0.7-1.7)^{\mathrm{a}}$ \\
\hline & Hiyama, 2002 & $117 / 116$ & Japanese & Hospital & *Pro vs Arg/Arg & $1.1(0.6-1.8)^{\mathrm{a}}$ \\
\hline & & & & & $\begin{array}{l}\text { Diffuse } \\
\text { Intestinal }\end{array}$ & $2.1(1.0-4.6)^{\mathrm{a}}$ \\
\hline & Zhang, 2003 & $120 / 277$ & British & Hospital & *Pro vs Arg/Arg & $\begin{array}{l}0.7(0.4-1.3)^{\mathrm{a}} \\
0.7(0.5-1.1)^{\mathrm{a}}\end{array}$ \\
\hline & & & & & Diffuse & $0.9(0.5-1.6)^{\mathrm{a}}$ \\
\hline & & & & & Intestinal & $0.6(0.4-1.1)^{\mathrm{a}}$ \\
\hline & Shen, 2004 & $324 / 317$ & Chinese & Population & Pro/Pro vs *Arg & $0.7(0.5-1.1)^{\mathrm{a}}$ \\
\hline & Wu, 2004 & $89 / 192$ & Chinese & Hospital & Pro/Pro vs Arg/Arg & $1.3(0.6-3.2)$ \\
\hline & $X i, 2004$ & $48 / 288$ & Chinese & Population & *Pro vs Arg/Arg & $1.6(0.8-3.1)^{a}$ \\
\hline & Lai, 2005 & $51 / 59$ & Chinese & Hospital & *Pro vs Arg/Arg & $1.4(0.6-2.9)^{\mathrm{a}}$ \\
\hline & & & & (10) & Diffuse & $2.0(0.8-5.0)^{\mathrm{a}}$ \\
\hline & & & & & Intestinal & $0.6(0.2-1.8)^{\mathrm{a}}$ \\
\hline & Lai, 2005 & $123 / 126$ & Chinese & Hospital & *Pro vs Arg/Arg & $1.3(0.8-2.2)^{\mathrm{a}}$ \\
\hline & Perez-P, 2005 & $65 / 182$ & Mexican & Hospital & *Pro vs Arg/Arg & $0.5(0.3-0.9)^{\mathrm{a}}$ \\
\hline & $\mathrm{Mu}, 2005$ & $206 / 415$ & Chinese & Population & *Pro vs Arg/Arg & $1.2(0.7-2.0)$ \\
\hline & Khayat, 2005 & $54 / 54$ & Brazil & Hospital & *Pro vs *Arg & $1.2(0.5-2.7)^{\mathrm{a}}$ \\
\hline & Belyavskaya, 2006 & $30 / 125$ & Russian & Hospital & *Pro vs Arg/Arg & $0.6(0.3-1.4)^{\mathrm{a}}$ \\
\hline & & & & & Diffuse & $0.2(0.1-1.1)^{\mathrm{a}}$ \\
\hline & & & & & Intestinal & $0.5(0.1-1.9)^{\mathrm{a}}$ \\
\hline & Chung, 2006 & $84 / 43$ & Korean & Hospital & *Pro vs Arg/Arg & $1.1(0.6-2.0)^{\mathrm{a}}$ \\
\hline & & & & & Diffuse & $0.7(0.3-1.8)^{\mathrm{a}}$ \\
\hline & & & & & Intestinal & $2.1(0.9-5.0)^{\mathrm{a}}$ \\
\hline & Yi, 2006 & $292 / 216$ & Korean & Hospital & *Pro vs Arg/Arg & $1.3(0.9-1.9)^{\mathrm{a}}$ \\
\hline & & & & & Diffuse & $1.3(0.9-2.0)^{\mathrm{a}}$ \\
\hline & Sul, 2006 & $155 / 134$ & American & Hospital & $\begin{array}{l}\text { Intestinal } \\
\text { Pro/Pro vs *Arg }\end{array}$ & $1.3(0.9-2.1)^{\mathrm{a}}$ \\
\hline TP53BP2 & & & & & Pro/Pro vs *Arg & $1.0(0.5-2.1)$ \\
\hline $\mathrm{rs} 1538140 \mathrm{C}>\mathrm{T}$ & & & & & CC vs*T & $1.5(1.1-2.1)$ \\
\hline rs1982610 A > T & & & & & AA vs*T & $1.5(1.0-2.1)$ \\
\hline rs1222158 G>A & & & & & GG $v s{ }^{*} \mathrm{~A}$ & $1.6(1.1-2.2)$ \\
\hline rs $2242188 \mathrm{~A}>\mathrm{T}$ & Ju, 2005 & $233 / 390$ & Korean & Hospital & $\mathrm{AA} v s^{*} \mathrm{~T}$ & $1.5(1.0-2.2)$ \\
\hline rs745697 G>T & & & & & GG vs*T & $1.0(0.7-1.5)$ \\
\hline rs1153942 G > T & & & & & $\pi v s * \mathrm{G}$ & $1.2(0.6-2.2)$ \\
\hline rs1222155 G>A & & & & & GG $v s^{*} \mathrm{~A}$ & $1.1(0.8-1.6)$ \\
\hline MDM2 309T > G (rs2279744) & Ohmiya, 2006 & $410 / 438$ & Japanese & Population & GG $v s * T$ & $1.5(1.0-2.1)$ \\
\hline & Cho, 2008 & 239/299 & Korean & Hospital & GG vs TT & $0.8(0.5-1.5)$ \\
\hline & & & & & Diffuse & $0.7(0.4-1.3)^{\mathrm{a}}$ \\
\hline & & & & & Intestinal & $0.8(0.4-1.3)^{\mathrm{a}}$ \\
\hline$p 73$ exon $24 \mathrm{G}>\mathrm{A}$ & Hamajima, 2002 & $144 / 241$ & Japanese & Hospital & GG vs AA & $0.7(0.3-1.9)^{\mathrm{a}}$ \\
\hline & Zhang, 2008 & $385 / 412$ & Chinese & Hospital & GG vs AA & $1.7(1.2-2.5)$ \\
\hline & & & & & $\begin{array}{l}\text { Diffuse } \\
\text { Intestinal }\end{array}$ & $\begin{array}{l}1.3(1.2-2.8) \\
1.1(0.6-2.3)\end{array}$ \\
\hline (b) Other cell cycle and apoptosis regu & & & & & & \\
\hline Cyclin D1 870G >A (rs603965) & Zhang, 2003 & $87 / 183$ & Chinese & Hospital & AA vs *G Cardia & $1.5(0.9-3.1)$ \\
\hline & Geddert, 2005 & $286 / 253$ & German & Hospital & $\mathrm{AA} v s{ }^{*} \mathrm{C}$ & $0.7(0.4-1.0)^{\mathrm{a}}$ \\
\hline & Song, 2007 & $253 / 442$ & Korean & Hospital & $\mathrm{AA} v{ }^{*} \mathrm{G}$ & $0.8(0.6-1.2)^{\mathrm{a}}$ \\
\hline & Jia, 2008 & $159 / 162$ & Chinese & Hospital & AA vs GG non-cardia & $0.3(0.2-0.7)$ \\
\hline p16 540C > G (rs11515) & Lai, 2005 & $123 / 119$ & Chinese & Hospital & ${ }^{*} \mathrm{G}$ vs CC & $0.4(0.1-1.9)^{\mathrm{a}}$ \\
\hline & Geddert, 2005 & $267 / 230$ & German & Hospital & ${ }^{*} \mathrm{G}$ vs CC & $0.8(0.5-1.3)^{\mathrm{a}}$ \\
\hline$p 21^{\text {WAF1/CIP1 }}$ Arg31Ser (rs1801270) & Wu, 2004 & $89 / 192$ & Chinese & Hospital & *Ser vs Arg/Arg & $1.1(0.5-2.2)$ \\
\hline & Xie, 2004 & $30 / 45$ & Chinese & Hospital & ${ }^{*}$ Ser vs Arg/Arg & $3.4(1.0-13.8)^{\mathrm{a}}$ \\
\hline & $\mathrm{Xi}, 2004$ & $48 / 288$ & Chinese & Population & Ser/Ser vs Arg/Arg Intestinal & $1.7(0.7-3.9)$ \\
\hline & Lai, 2005 & $123 / 119$ & Chinese & Hospital & *Ser vs Arg/Arg & $0.7(0.4-1.2)^{\mathrm{a}}$ \\
\hline 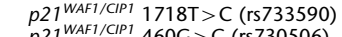 & $X i, 2004$ & $48 / 288$ & Chinese & Population & CC vs TT & $1.7(0.7-3.9)^{\mathrm{a}}$ \\
\hline p21 & & & & & CC vs GG & $1.6(0.7-3.9)^{\mathrm{a}}$ \\
\hline H-RAS T81C (rs12628) & Zhang, 2008 & $90 / 448$ & Chinese & Population & ${ }^{*} \mathrm{C}$ vs TT & $3.7(2.2-6.0)$ \\
\hline L-myc EcoR1 & Ishizaki, 1990 & $60 / 100$ & Japanese & Hospital & ${ }^{*} \mathrm{~S} v s \mathrm{LL}$ & $0.7(0.3-1.5)^{\mathrm{a}}$ \\
\hline & Kato, 1996 & $82 / 151$ & Japanese & Hospital & *S vs LL & \\
\hline & & & & & Diffuse & $1.2(0.4-3.4)$ \\
\hline & & & & & Intestinal & $1.4(0.5-4.0)$ \\
\hline & Kato, 1997 & $284 / 284$ & Japanese & Hospital & LS vs LL & $1.6(1.0-2.3)$ \\
\hline & Shibuta, 1998 & $61 / 107$ & Japanese & Hospital & *S vs LL & $3.1(1.3-7.2)$ \\
\hline & & & & & Diffuse & $2.9(0.9-10.2)^{\mathrm{a}}$ \\
\hline & & & & & Intestinal & $2.8(0.9-10.0)^{\mathrm{a}}$ \\
\hline & Isbir, 2002 & $25 / 83$ & Turkish & Hospital & ${ }^{*} \mathrm{~S}$ vs LL & $4.6(1.5-16.8)^{\mathrm{a}}$ \\
\hline & Dlugosz, 2002 & $100 / 65$ & Caucasian & Hospital & *S vs LL & $0.5(0.2-1.1)^{a}$ \\
\hline & & & & & Diffuse & $0.6(0.2-1.4)^{\mathrm{a}}$ \\
\hline & & & & & Intestinal & $0.4(0.2-1.1)^{\mathrm{a}}$ \\
\hline & Nan, 2005 & $110 / 220$ & Chinese & Hospital & *S vs LL & $1.6(0.9-2.9)^{\mathrm{a}}$ \\
\hline Survivin -31G > C (rs17884799) & Cheng, 2008 & $96 / 67$ & Chinese & Hospital & CC vs GG & $4.8(2.9-8.0)$ \\
\hline & Yang, 2008 & $220 / 220$ & Chinese & Hospital & ${ }^{*} \mathrm{C}$ vs GG & $1.4(0.9-2.2)$ \\
\hline & & & & & Cardia & $1.0(0.5-1.9)$ \\
\hline & & & & & Non-cardia & $2.0(1.2-3.3)$ \\
\hline$D R 4626 \mathrm{C}>\mathrm{G}(\mathrm{rs} 4871857)$ & Kuraoka, 2005 & $274 / 344$ & Japanese & Hospital & ${ }^{*} \mathrm{C}$ vs CC & $1.1(0.6-2.0)^{\mathrm{a}}$ \\
\hline Erf3 GGC VNTR & Brito, 2005 & $278 / 200$ & Portuguese & Hospital & $* 10$ vs *12 & $19.9(1.2-333.9)$ \\
\hline KLF6 -27G > A (rs3750861) & Cho, 2008 & $264 / 299$ & Korean & Hospital & AA vs GG & $9.5(1.7-52.3)$ \\
\hline PPAR $\gamma$ Pro12Ala (rs1801282) & Liao, 2006 & 104/104 & Chinese & Hospital & *Ala vs Pro/Pro Non-cardia & $2.5(1.1-5.8)$ \\
\hline & Tahara, 2007 & $215 / 201$ & Japanese & Hospital & Pro/Ala vs Pro/Pro & $2.4(1.0-5.7)$ \\
\hline & & & & & Cardia & $5.0(0.5-51.4)$ \\
\hline & & & & & Non-cardia & $2.4(1.0-5.7)$ \\
\hline & & & & & Diffuse & $2.2(0.8-6.2)$ \\
\hline & & & & & Intestinal & $2.9(1.1-7.7)$ \\
\hline & Prasad, 2008 & $62 / 241$ & Indian & Hospital & *Ala vs Pro/Pro & $2.1(1.1-4.1)$ \\
\hline RUNX3 364C>T & $\mathrm{Hu}, 2005$ & $178 / 361$ & Chinese & Hospital & $\pi$ vs CC & $1.1(0.4-2.8)$ \\
\hline LAPTM $4 B$ VNTR in & Liu, 2007 & $214 / 350$ & Chinese & Hospital & ${ }^{*} 1$ vs *2/2 & $2.4(1.2-4.8)$ \\
\hline $5^{\prime}$ UTR & & & & & & \\
\hline
\end{tabular}

Abbreviations: $\mathrm{Cl}$, confidence interval; GC, gastric cancer; OR, odds ratio; VNTR, variable number tandem repeat.

Allele carrier.

${ }^{a}$ Calculated based on genotype or allele frequency extracted from corresponding article. 
Table 2 Association between growth factors gene polymorphisms and gastric cancer susceptibility

\begin{tabular}{|c|c|c|c|c|c|c|}
\hline \multirow[b]{2}{*}{ Polymorphism (SNP ID) } & \multirow[b]{2}{*}{$\begin{array}{l}\text { First author, } \\
\text { publish year }\end{array}$} & \multicolumn{3}{|c|}{ Study design and population } & \multicolumn{2}{|c|}{ Association with GC } \\
\hline & & $\begin{array}{l}\text { No. of cases/ } \\
\text { controls }\end{array}$ & Population & Setting & Groups compared & OR $(95 \% \mathrm{Cl})$ \\
\hline \multirow{4}{*}{$\begin{array}{l}\text { EGF 5'UTR } 61 \mathrm{~A}>\mathrm{G} \\
(\mathrm{rs} 4444903)\end{array}$} & Hamai, 2005 & $200 / 230$ & Japanese & Hospital & ${ }^{*} \mathrm{~A}$ vs GG & $0.6(0.4-0.9)$ \\
\hline & & & & & $\begin{array}{l}\text { Diffuse } \\
\text { Intestinal }\end{array}$ & $\begin{array}{l}0.8(0.5-1.4)^{\mathrm{a}} \\
0.5(0.3-0.7)^{\mathrm{a}}\end{array}$ \\
\hline & Goto, 2005 & $202 / 454$ & Japanese & Hospital & ${ }^{*} \mathrm{~A}$ vs GG & $1.0(0.7-1.4)$ \\
\hline & Jin, 2007 & $617 / 660$ & Chinese & Population & ${ }^{*} \mathrm{~A}$ vs GG & $0.8(0.6-1.0)$ \\
\hline \multirow{4}{*}{$\begin{array}{l}E G F-1380 G>A(r s 11568835) \\
E G F-1744 A>G(r s 3756261) \\
H E R 2 \text { lle655Val (rs1801200) } \\
T G F B 1-509 C>T(r s 1800469)\end{array}$} & Jin, 2007 & $617 / 660$ & Chinese & Population & $\begin{array}{l}\text { AA vs GG } \\
\text { GG vs AA }\end{array}$ & $\begin{array}{l}0.9(0.5-1.7) \\
1.2(0.7-2.1)\end{array}$ \\
\hline & Kuraoka, 2003 & $212 / 287$ & Japanese & Hospital & Val/Val vs lle/lle & $3.3(1.1-9.8)$ \\
\hline & Jin, 2007 & $636 / 676$ & Chinese & Population & ${ }^{*} \mathrm{~T}$ vs $\mathrm{CC}$ & $0.7(0.5-0.8)$ \\
\hline & $\mathrm{Li}, 2008$ & $167 / 193$ & Chinese & Hospital & TT vs CC & $2.1(1.1-3.8)$ \\
\hline \multirow[t]{4}{*}{ TGFB1 869T > C (rs1982073) } & Jin, 2007 & $636 / 676$ & Chinese & Population & CC vs TT & $0.9(0.7-1.3)$ \\
\hline & García-G, 2006 & $142 / 342$ & Spanish & Hospital & CC vs TT & $1.1(0.6-1.8)^{\mathrm{a}}$ \\
\hline & García-G, 2007 & $404 / 404$ & Spanish & Hospital & CC vs TT & $0.9(0.6-1.1)$ \\
\hline & Li, 2008 & $167 / 193$ & Chinese & Hospital & CC vs TT & $4.0(2.1-7.8)$ \\
\hline \multirow[t]{2}{*}{ TGFB1 915G >C (rs1800471) } & García-G, 2006 & $142 / 342$ & Spanish & Hospital & ${ }^{*} \mathrm{C}$ vs GG & $1.2(0.7-2.2)^{a}$ \\
\hline & García-G, 2007 & $404 / 404$ & Spanish & Hospital & ${ }^{*} \mathrm{C}$ vs GG & $1.4(0.9-2.1)$ \\
\hline TGFBR2 -875G > A (rs3087465) & Jin, 2007 & $636 / 676$ & Chinese & Population & ${ }^{*} \mathrm{~A}$ vs GG & $0.7(0.5-0.9)$ \\
\hline INS $-23 \mathrm{~A}>\mathrm{T}(\mathrm{rs} 698)$ & Li, 2007 & $160 / 166$ & Chinese & Hospital & ${ }^{*} \mathrm{~T}$ vs AA & $1.4(0.8-2.7)$ \\
\hline IGF1R A > G (rs2229765) & & & & & GG vs AA & $1.2(0.7-2.3)$ \\
\hline IGF-II 17200A > G (rs680) & & & & & GG vs AA & $1.5(0.8-2.9)$ \\
\hline IGFBP1 643A > G (rs3793344) & & & & & GG vs AA & $1.4(0.7-2.6)$ \\
\hline \multirow[t]{2}{*}{ IGFBP3 $-202 \mathrm{~A}>C(\mathrm{rs} 2854744)$} & Zhang, 2004 & $120 / 267$ & British & Hospital & $C C$ vs *A & $2.1(1.1-3.9)^{\mathrm{a}}$ \\
\hline & Chen, 2008 & $576 / 647$ & Chinese & Hospital & CC vs AA & $1.3(0.7-2.1)$ \\
\hline IGFBP3 Gly32Ala (rs2854746) & Chen, 2008 & $576 / 647$ & Chinese & Hospital & Ala/Ala vs Gly/Gly & $2.4(1.5-3.9)$ \\
\hline$M K-2669 \mathrm{G}>\mathrm{A}(\mathrm{rs} 20542)$ & Lai, 2005 & $123 / 126$ & Chinese & Hospital & ${ }^{*} \mathrm{~A}$ vs GG & $1.3(0.5-3.1)^{\mathrm{a}}$ \\
\hline \multirow{2}{*}{ Pepsinogen C I/D } & Liu, 2003 & $73 / 42$ & Chinese & Hospital & ${ }^{*} 6 / 6$ vs others & $2.9(1.1-8.5)^{a}$ \\
\hline & Pinto-C, 2006 & $57 / 127$ & Portuguese & Population & $\begin{array}{l}{ }^{*} 6 \text { present vs } \\
\text { absent }\end{array}$ & $0.4(0.2-0.8)$ \\
\hline
\end{tabular}

Abbreviations: GC, gastric cancer; OR, odds ratio; UTR, untranslated region; I/D, insertion/deletion.

*Allele carrier.

${ }^{a}$ Calculated based on genotype or allele frequency extracted from corresponding article.

Recently, polymorphisms related with growth factors were assessed as potential risk markers of GC (Table 2). At 5' UTR in epidermal growth factor (EGF) gene, 61G carrier was associated with reduced GC risk in the Asian studies from Japan and China. ${ }^{56-58}$ Ile655Val variant of HER2, a member of the EGF receptor family, showed a significant association with GC. ${ }^{59}$ TGFB1-509T and TGFBR2-875A were reported determining high levels of transforming growth factor $\beta 1$ (TGF- $\beta 1$ ) and TGF- $\beta$ receptor 2 (TGF- $\beta$ R2), respectively. Decreased risks of GC were observed for these two alleles in one study from China. ${ }^{60} \mathrm{Li} \mathrm{ZQ}$ et al ${ }^{64}$ studied polymorphisms of insulin-like growth factors (IGF)-related genes. No association was found between GC risk and SNPs of insulin (INS), IGF-II, insulin-like growth factor binding protein 1 (IGFBP1) and IGF-1 receptor (IGF1R) genes. However, a significant association was observed for IGFBP3 $-202 A>C$ in a British population ${ }^{65}$ and for Gly32Ala in a Chinese population. ${ }^{64}$ Pepsinogen C (PGC) insertion/ deletion variant between exons 7 and 8 was significantly associated with GC risk in studies carried out among the Chinese and Portuguese populations in reverse directions. ${ }^{67,68}$

\section{Discussion}

This systematic review addressed the association of cell proliferation-related genetic polymorphisms with GC reported up to 15 September 2008. Twenty-three polymorphisms significantly related to GC in at least one published study were identified, which suggests that polymorphisms in genes implicated in cell proliferation could be candidate biomarkers of GC risk.

Cell cycle and apoptosis regulators, which are directly involved in the initiation of cellular malignant proliferation, have long been preferred targets as cancer risk markers. ${ }^{75}$ Our analysis regarding polymorphisms in TP53 are consistent with and extend findings from a recent meta-analysis focusing on the Arg73Pro polymorphism. ${ }^{76}$ Our meta-analysis, which included two additional studies on this polymorphism, ${ }^{19,21}$ confirmed associations to vary by population and histological type of GC. Significant heterogeneity was observed among all the included 14 studies on TP53 72Pro, with no evidence of an overall association with GC risk. When stratified by ethnicity, however, studies included in subgroup analyses displayed better homogeneity, with an indication of an increased risk 


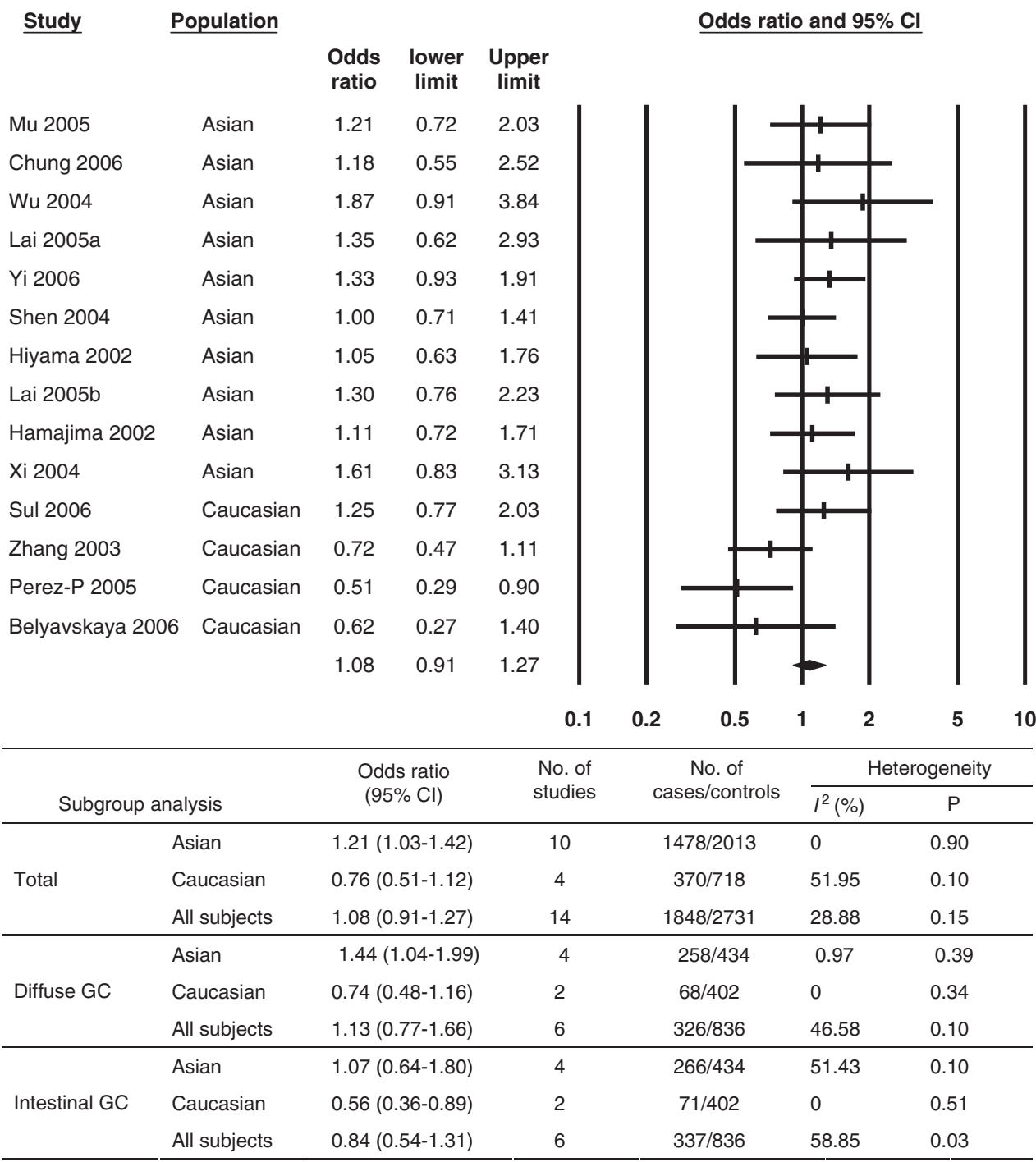

Figure 2 Meta-analysis of TP53 72Pro and gastric cancer susceptibility.

among the Arg allele carriers in Asians and a tentatively reduced risk among Caucasians. Such inverse associations might be explained by differing environmental factors, which act jointly with either apoptotic or DNA repair machinery, respectively. Environmental risk factors might also determine the mutational spectrum of TP53, which was also shown to contribute to the functional consequences of the Pro72Arg polymorphism. ${ }^{77}$ Alternatively, a different degree of linkage disequilibrium in different ethnicities of this variant with another functional variant might underlie the findings. One candidate polymorphism could be the 16-bp duplication in intron 3 that has previously been associated with reduced TP53 mRNA. $^{78}$

Heterogeneous results were found for the other two most widely studied polymorphisms in cell cycle-related genes, L-myc EcoRI polymorphism ${ }^{39-42}$ and $p 21$ Arg31-
Ser, ${ }^{18,19,21,37}$ even in the same ethnicity. Such an inconsistency may be partly explained by study design and the interaction with non-genetic risk factors, such as $H$. pylori infection and dietary factors, which strongly vary between populations. So, besides stratification by ethnicity and different type of GC, larger population-based studies with careful ascertainment of 'lifestyle' and 'environmental' factors are essential to fully understand the role of host genetic susceptibility.

A consistently increased risk was observed for PPAR- $\gamma$ 12Ala carriers in the Chinese, Japanese and Indian populations. ${ }^{51-53}$ PPAR- $\gamma$ is a member of the nuclear hormone receptor family that plays an important role in cell differentiation and regulation of metabolism. A potential interplay between PPAR- $\gamma$ Pro12Ala polymorphism and $H$. pylori infection was observed in the 


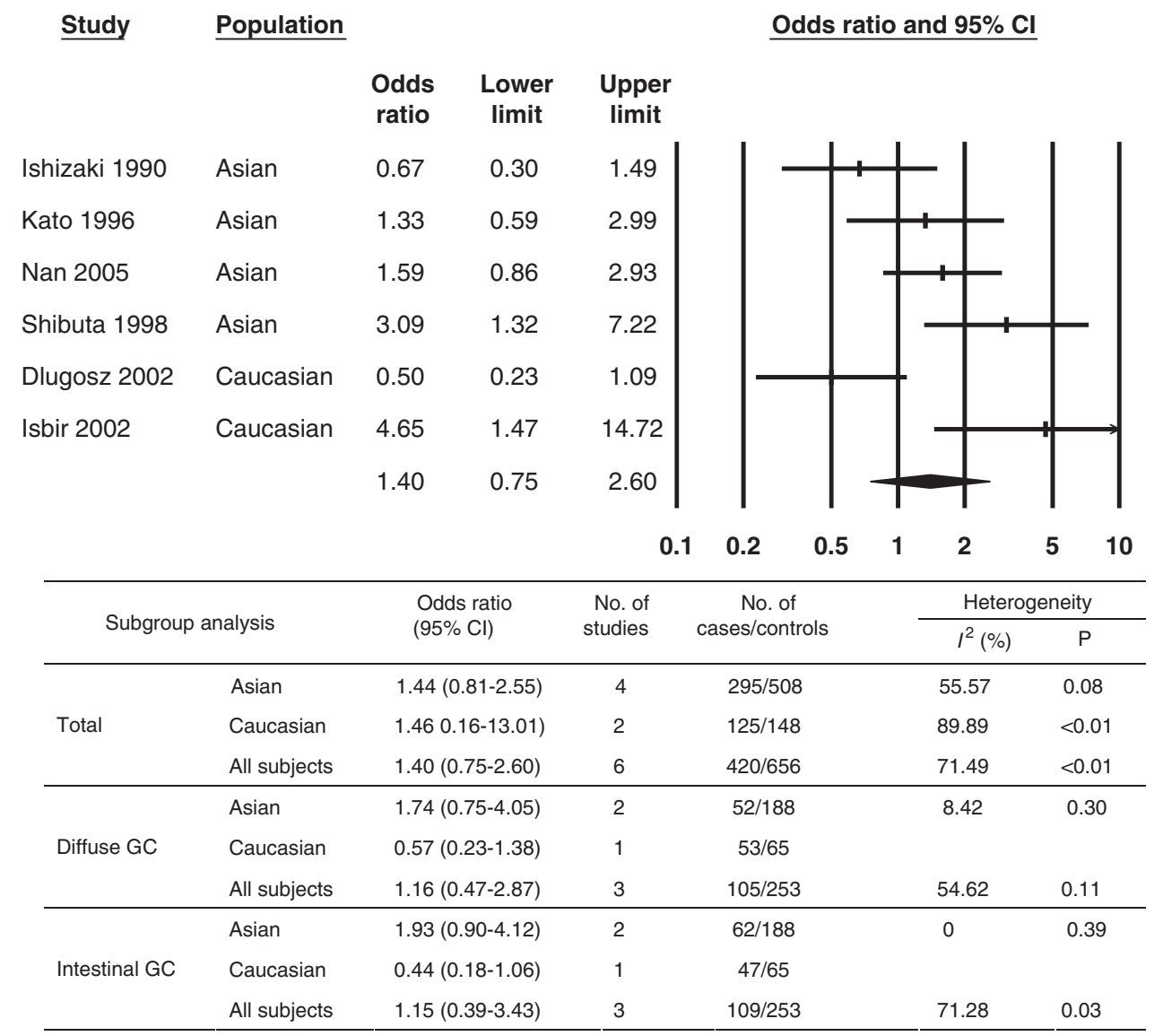

Figure 3 Meta-analysis of L-myc S allele and gastric cancer susceptibility.

development of GC. ${ }^{51,53}$ It was also suggested that this polymorphism may be associated with gastric mucosal atrophy in $H$. pylori-infected patients, thereby increasing the risk of GC. ${ }^{52}$ Further studies on different ethnic groups are needed to confirm the observed association and to clarify the role of PPAR- $\gamma$ during gastric carcinogenesis.

Polymorphisms determining higher level of growth factors and related receptors, which are important to tissue repair, were associated with reduced risk of GC. Such associations were observed for $E G F 5^{\prime}$ UTR $61 \mathrm{G}>\mathrm{A},{ }^{56,58}$ TGFB1 $-509 \mathrm{C}>\mathrm{T},{ }^{60}$ TGFBR2 $-875 \mathrm{G}>\mathrm{A},{ }^{60}$ and IGFBP3 $-202 \mathrm{~A}>\mathrm{C}^{65}$ and Gly32Ala. ${ }^{66}$ PGC was reported to not only act as a digestive enzyme, but might also be a growth factor during the healing of gastric lesions. ${ }^{79}$ Genetic polymorphisms in PGC gene determining lower expression were also supposed to contribute to gastric ulcer and GC by failing to prevent disease development. ${ }^{68,80}$

Although this review indicates that cell proliferationrelated genetic polymorphisms could be candidate biomarkers in GC risk, their overall effect seems to be modest and results were often inconsistent. Our analyses suggest that the inconsistencies may be explained, in part, by differences between the study populations and potentially different effects on different types of GC. In addition, different covariates were considered and controlled in different studies as presented in the Supplementary Tables. Considering $H$. pylori infection is suggested to be a (close to) necessary condition for development of noncardia gastric cancer, ${ }^{81,82}$ the role of genetic polymorphisms may primarily be restricted to some (minor) modulation of the risk in the presence of $H$. pylori infection. In consideration of the potential misclassification of $H$. pylori status due to disease-related clearance of infection, ${ }^{83}$ reports on gene$H$. pylori interaction should be interpreted with caution.

There are some limitations to this systematic review that need careful consideration. First, because of the limited information supplied by included studies and the small sample sizes, relevant stratifications (eg, by ethnicity or type of GC) could not be made for many studies. Second, some of the included studies did not mention whether polymorphisms in controls were in Hardy-Weinberg equilibrium. However, no significant deviation from Hardy-Weinberg equilibrium was observed for all included studies based on our own calculations (data not shown), except the study by Xie et $a l,{ }^{37}$ which presented allele frequencies only. Third, owing to the heterogeneity in 
length and detail of presentation of the included studies, no consistent formal rating of quality of studies was possible. Fourth, observed associations in the meta-analyses were generally weak and may partly reflect false positive results due to multiple testing.

In conclusion, this systematic review suggested that cell proliferation-related genetic polymorphisms could be candidate biomarkers of GC risk, but current evidence for the use for risk stratification is still very limited. Stratifications by ethnicity and GC type seem to be crucial in future studies aiming to clarify the effect of genetic polymorphisms on GC risk.

\section{Acknowledgements}

The study was funded in part by a grant from the BadenWuerttemberg State Ministry of Science, Research and Arts. The writing of this paper was funded in part by a scholarship from the German Academic Exchange Service (DAAD) to $L G$.

\section{References}

1 Forman D, Burley VJ: Gastric cancer: global pattern of the disease and an overview of environmental risk factors. Best Pract Res Clin Gastroenterol 2006; 20: 633-649.

2 Tsugane S: Salt, salted food intake, and risk of gastric cancer: epidemiologic evidence. Cancer Sci 2005; 96: 1-6.

3 Murakami K, Kodama M, Fujioka T: Latest insights into the effects of Helicobacter pylori infection on gastric carcinogenesis. World J Gastroenterol 2006; 12: 2713-2720.

4 Kelley JR, Duggan JM: Gastric cancer epidemiology and risk factors. J Clin Epidemiol 2003; 56: 1-9.

5 El-Omar EM, Carrington M, Chow WH et al: Interleukin-1 polymorphisms associated with increased risk of gastric cancer. Nature 2000; 404: 398-402.

6 Gonzalez CA, Sala N, Capella G: Genetic susceptibility and gastric cancer risk. Int J Cancer 2002; 100: 249-260.

7 Silva F, Carvalho F, Peixoto A et al: MUC1 gene polymorphism in the gastric carcinogenesis pathway. Eur J Hum Genet 2001; 9: $548-552$.

8 Zhang J, Dou C, Song Y et al: Polymorphisms of tumor necrosis factor-alpha are associated with increased susceptibility to gastric cancer: a meta-analysis. J Hum Genet 2008; 53: 479-489.

9 Wang GY, Lu CQ, Zhang RM, Hu XH, Luo ZW: The E-cadherin gene polymorphism 160C- $>$ A and cancer risk: a huge review and meta-analysis of 26 case-control studies. Am J Epidemiol 2008; 167: $7-14$

10 Sun L, Sun YH, Wang B, Cao HY, Yu C: Methylenetetrahydrofolate reductase polymorphisms and susceptibility to gastric cancer in Chinese populations: a meta-analysis. Eur J Cancer Prev 2008; 17: 446-452.

11 Pai M, McCulloch M, Gorman JD et al: Systematic reviews and meta-analyses: an illustrated, step-by-step guide. Natl Med J India 2004; 17: 86-95.

12 Moher D, Cook DJ, Eastwood S, Olkin I, Rennie D, Stroup DF: Improving the quality of reports of meta-analyses of randomised controlled trials: the QUOROM statement. Quality of reporting of meta-analyses. Lancet 1999; 354: 1896-1900.

13 Gao L, Nieters A, Brenner H: Meta-analysis: tumour invasionrelated genetic polymorphisms and gastric cancer susceptibility. Aliment Pharmacol Ther 2008; 28: 565-573.

14 Hamajima N, Matsuo K, Suzuki T et al: No associations of p73 G4C14-to-A4T14 at exon 2 and p53 Arg72Pro polymorphisms with the risk of digestive tract cancers in Japanese. Cancer Lett 2002; 181: 81-85.
15 Hiyama T, Tanaka S, Kitadai Y et al: p53 Codon 72 polymorphism in gastric cancer susceptibility in patients with Helicobacter pylori-associated chronic gastritis. Int J Cancer 2002; 100: 304-308.

16 Zhang ZW, Newcomb P, Hollowood A et al: Age-associated increase of codon 72 Arginine p53 frequency in gastric cardia and non-cardia adenocarcinoma. Clin Cancer Res 2003; 9: $2151-2156$

17 Shen H, Solari A, Wang X et al: P53 codon 72 polymorphism and risk of gastric cancer in a Chinese population. Oncol Rep 2004; 11: $1115-1120$

$18 \mathrm{Wu}$ MT, Chen MC, Wu DC: Influences of lifestyle habits and p53 codon 72 and $\mathrm{p} 21$ codon 31 polymorphisms on gastric cancer risk in Taiwan. Cancer Lett 2004; 205: 61-68.

19 Xi YG, Ding KY, Su XL et al: p53 polymorphism and p21WAF1/ CIP1 haplotype in the intestinal gastric cancer and the precancerous lesions. Carcinogenesis 2004; 25: 2201-2206.

20 Lai KC, Chen WC, Tsai FJ, Li SY, Jeng LB: Arginine and proline alleles of the p53 gene are associated with different locations of gastric cancer. Hepatogastroenterology 2005; 52: 944-948.

21 Lai KC, Chen WC, Jeng LB, Li SY, Chou MC, Tsai FJ: Association of genetic polymorphisms of MK, IL-4, p16, p21, p53 genes and human gastric cancer in Taiwan. Eur J Surg Oncol 2005; 31: 1135 1140.

22 Perez-Perez GI, Bosques-Padilla FJ, Crosatti ML, Tijerina-Menchaca R, Garza-Gonzalez E: Role of p53 codon 72 polymorphism in the risk of development of distal gastric cancer. Scand $J$ Gastroenterol 2005; 40: 56-60.

$23 \mathrm{Mu} \mathrm{LN}, \mathrm{Lu}$ QY, Yu SZ et al: Green tea drinking and multigenetic index on the risk of stomach cancer in a Chinese population. Int J Cancer 2005; 116: 972-983.

24 Khayat AS, Lobo GL, Moura LE et al: Polymorphisms of the TP53 codon 72 and WRN codon 1367 in individuals from Northern Brazil with gastric adenocarcinoma. Clin Exp Med 2005; 5: $161-168$.

25 Belyavskaya VA, Vardosanidze VK, Smirnova OY et al: Genetic status of p53 in stomach cancer: somatic mutations and polymorphism of codon 72. Bull Exp Biol Med 2006; 141: 243-246.

26 Chung WC, Lee KM, Lee BI et al: P53 genetic polymorphism of gastric cancer in Korea. Korean J Intern Med 2006; 21: 28-32.

27 Yi SY, Lee WJ: A p53 genetic polymorphism of gastric cancer: difference between early gastric cancer and advanced gastric cancer. World J Gastroenterol 2006; 12: 6536-6539.

28 Sul J, Yu GP, Lu QY et al: P53 codon 72 polymorphisms: a casecontrol study of gastric cancer and potential interactions. Cancer Lett 2006; 238: 210-223.

$29 \mathrm{Ju} \mathrm{H}$, Lee KA, Yang M et al: TP53BP2 locus is associated with gastric cancer susceptibility. Int J Cancer 2005; 117: 957-960.

30 Ohmiya N, Taguchi A, Mabuchi N et al: MDM2 promoter polymorphism is associated with both an increased susceptibility to gastric carcinoma and poor prognosis. J Clin Oncol 2006; 24: $4434-4440$

31 Cho YG, Choi BJ, Song JH et al: No association of MDM2 T309G polymorphism with susceptibility to Korean gastric cancer patients. Neoplasma 2008; 55: 256-260.

32 Zhang WH, Wang XD, Xie XD et al: Association of the p73 gene G4C14-to-A4T14 polymorphism with increased gastric cancer risk in a northwestern Chinese population. Chin J Clin Oncol 2008; 5: 157-160.

33 Zhang J, Li Y, Wang R et al: Association of cyclin D1 (G870A) polymorphism with susceptibility to esophageal and gastric cardiac carcinoma in a northern Chinese population. Int J Cancer 2003; 105: 281-284.

34 Geddert H, Kiel S, Zotz RB et al: Polymorphism of p16 INK4A and cyclin D1 in adenocarcinomas of the upper gastrointestinal tract. J Cancer Res Clin Oncol 2005; 131: 803-808.

35 Song JH, Kim CJ, Cho YG et al: Association of cyclin D1 G870A polymorphism with susceptibility to gastric cancers in Korean male patients. Neoplasma 2007; 54: 235-239. 
36 Jia A, Gong J, Li Y et al: GG genotype of cyclin D1 G870A polymorphism is associated with non-cardiac gastric cancer in a high-risk region of China. Scand J Gastroenterol 2008; 43: $1353-1359$.

37 Xie HL, Su Q, He XS et al: Expression of p21(WAF1) and p53 and polymorphism of p21(WAF1) gene in gastric carcinoma. World $J$ Gastroenterol 2004; 10: 1125-1131.

38 Zhang Y, Jin M, Liu B et al: Association between H-RAS T81C genetic polymorphism and gastrointestinal cancer risk: a population based case-control study in China. BMC Cancer 2008; 8: 256.

39 Ishizaki K, Kato M, Ikenaga M, Honda K, Ozawa K, Toguchida J: Correlation of L-myc genotypes to metastasis of gastric cancer and breast cancer. J Natl Cancer Inst 1990; 82: 238-239.

40 Kato S, Onda M, Matsukura N et al: Genetic polymorphisms of the cancer related gene and Helicobacter pylori infection in Japanese gastric cancer patients. An age and gender matched case-control study. Cancer 1996; 77: 1654-1661.

41 Kato S, Onda M, Matsukura N et al: Helicobacter pylori infection and genetic polymorphisms for cancer-related genes in gastric carcinogenesis. Biomed Pharmacother 1997; 51: 145-149.

42 Shibuta K, Mori M, Haraguchi M, Yoshikawa K, Ueo H, Akiyoshi $\mathrm{T}$ : Association between restriction fragment length polymorphism of the L-myc gene and susceptibility to gastric cancer. Br J Surg 1998; 85: 681-684.

43 Isbir T, Yaylim I, Arikan S et al: Close correlation between restriction fragment length polymorphism of L-myc gene and susceptibility to gastric cancer. Cancer Detect Prev 2002; 26: $454-457$.

44 Dlugosz A, Adler G, Ciechanowicz A, Jaroszewicz-Heigelmann H, Starzynska T: EcoRI polymorphism of the L-myc gene in gastric cancer patients. Eur J Gastroenterol Hepatol 2002; 14: $1231-1235$.

45 Nan HM, Song YJ, Yun HY, Park JS, Kim H: Effects of dietary intake and genetic factors on hypermethylation of the hMLH1 gene promoter in gastric cancer. World J Gastroenterol 2005; 11: $3834-3841$.

46 Cheng ZJ, Hu LH, Huang SJ: Correlation of $-31 \mathrm{G} / \mathrm{C}$ polymorphisms of survivin promoter to tumorigenesis of gastric carcinoma. Ai Zheng 2008; 27: 258-263. Chinese.

47 Yang L, Zhu H, Zhou B et al: The association between the survivin C-31G polymorphism and gastric cancer risk in a Chinese population. Dig Dis Sci 2008; 54: 1021-1028.

48 Kuraoka K, Matsumura S, Sanada Y et al: A single nucleotide polymorphism in the extracellular domain of TRAIL receptor DR4 at nucleotide 626 in gastric cancer patients in Japan. Oncol Rep 2005; 14: 465-470.

49 Brito M, Malta-Vacas J, Carmona B et al: Polyglycine expansions in eRF3/GSPT1 are associated with gastric cancer susceptibility. Carcinogenesis 2005; 26: 2046-2049.

50 Cho YG, Lee HS, Song JH et al: KLF6 IVS1 -27G/A polymorphism with susceptibility to gastric cancers in Korean. Neoplasma 2008; 55: $47-50$

51 Liao SY, Zeng ZR, Leung WK et al: Peroxisome proliferatoractivated receptor-gamma Pro12Ala polymorphism, Helicobacter pylori infection and non-cardia gastric carcinoma in Chinese. Aliment Pharmacol Ther 2006; 23: 289-294.

52 Tahara T, Arisawa T, Shibata T et al: Influence of peroxisome proliferator-activated receptor (PPAR)gamma Plo12Ala polymorphism as a shared risk marker for both gastric cancer and impaired fasting glucose (IFG) in Japanese. Dig Dis Sci 2008; 53: $614-621$.

53 Prasad KN, Saxena A, Ghoshal UC, Bhagat MR, Krishnani N: Analysis of Pro12Ala PPAR gamma polymorphism and Helicobacter pylori infection in gastric adenocarcinoma and peptic ulcer disease. Ann Oncol 2008; 19: 1299-1303.

$54 \mathrm{Hu}$ S, Song QB, Ke YH, Hu PJ, Ceng ZR: Relationship between RUNX3 gene 364 locus $\mathrm{C} \rightarrow \mathrm{T}$ mutation and gastric cancer in Chinese. Chin J Pathophysiol 2005; 21: 1905-1908. Chinese.
55 Liu Y, Zhang QY, Qian N, Zhou RL: Relationship between LAPTM4B gene polymorphism and susceptibility of gastric cancer. Ann Oncol 2007; 18: 311-316.

56 Hamai $\mathrm{Y}$, Matsumura S, Matsusaki $\mathrm{K}$ et al: A single nucleotide polymorphism in the $5^{\prime}$ untranslated region of the EGF gene is associated with occurrence and malignant progression of gastric cancer. Pathobiology 2005; 72: 133-138.

57 Goto Y, Ando T, Goto H, Hamajima N: No association between EGF gene polymorphism and gastric cancer. Cancer Epidemiol Biomarkers Prev 2005; 14: 2454-2456.

58 Jin G, Miao R, Deng Y et al: Variant genotypes and haplotypes of the epidermal growth factor gene promoter are associated with a decreased risk of gastric cancer in a high-risk Chinese population. Cancer Sci 2007; 98: 864-868.

59 Kuraoka K, Matsumura S, Hamai Y et al: A single nucleotide polymorphism in the transmembrane domain coding region of HER-2 is associated with development and malignant phenotype of gastric cancer. Int J Cancer 2003; 107: 593-596.

60 Jin G, Wang L, Chen W et al: Variant alleles of TGFB1 and TGFBR2 are associated with a decreased risk of gastric cancer in a Chinese population. Int J Cancer 2007; 120: 1330-1335.

$61 \mathrm{Li} \mathrm{T}$, Cao BW, Dai Y, Cui H, Yang HL, Xu CQ: Correlation of transforming growth factor beta-1 gene polymorphisms C-509T and $\mathrm{T} 869 \mathrm{C}$ and the risk of gastric cancer in China. J Gastroenterol Hepatol 2008; 23: 638-642.

62 Garcia-Gonzalez MA, Strunk M, Piazuelo E et al: TGFB1 gene polymorphisms: their relevance in the susceptibility to Helicobacter pylori-related diseases. Genes Immun 2006; 7: $640-646$.

63 Garcia-Gonzalez MA, Lanas A, Quintero E et al: Gastric cancer susceptibility is not linked to pro-and anti-inflammatory cytokine gene polymorphisms in whites: a Nationwide Multicenter Study in Spain. Am J Gastroenterol 2007; 102: $1878-1892$.

$64 \mathrm{Li} \mathrm{ZQ}, \mathrm{Yu}$ WP, Xie XD et al: Association of gastric cancer with tyrosine hydroxylase gene polymorphism in a northwestern Chinese population. Clin Exp Med 2007; 7: 98-101.

65 Zhang ZW, Newcomb PV, Moorghen M et al: Insulin-like growth factor binding protein-3: relationship to the development of gastric pre-malignancy and gastric adenocarcinoma (United Kingdom). Cancer Causes Control 2004; 15: $211-218$.

66 Chen W, Wang L, Ke Q et al: The role of IGFBP3 functional polymorphisms in the risk of gastric cancer in a high-risk Chinese population. Eur J Cancer Prev 2008; 17: 82-87.

67 Liu HJ, Guo XL, Dong M, Wang L, Yuan Y: Association between pepsinogen $\mathrm{C}$ gene polymorphism and genetic predisposition to gastric cancer. World J Gastroenterol 2003; 9: 50-53.

68 Pinto-Correia AL, Sousa H, Fragoso M et al: Gastric cancer in a Caucasian population: role of pepsinogen $\mathrm{C}$ genetic variants. World J Gastroenterol 2006; 12: 5033-5036.

69 Zhang ZW, Newcomb P, Hollowood A et al: A comparison study of gastric cancer risk in patients with duodenal and gastric ulcer: roles of gastric mucosal histology and p53 codon 72 polymorphism. Dig Dis Sci 2004; 49: 254-259.

70 Tahara T, Arisawa T, Shibata T, Yuwang F, Hirata I, Nakano H: Peroxisome proliferator-activated receptor gamma Pro12Ala polymorphism influences the susceptibility of a Japanese population to gastric cancer. Scand J Gastroenterol 2009; 44: 512.

71 Liao SY, Ceng ZR, Zhou SZ, Chen B, Hu PJ: Association of Pro12Ala polymorphism in the peroxisome proliferator-activated receptor-gamma2 with gastric cancers in China. Chin J Pathophysiol 2007; 23: 757-760. Chinese.

$72 \mathrm{Mu}$ LN, Zhou XF, Ding BG et al: Genetic polymorphisms of p53 codon72 and the risk of gastric cancer-case-control study. China Oncology 2003; 13: 1-4. Chinese. 
73 Xie HL, Su Q, He XS et al: Relationship between p21WAF1 gene polymorphism and Carcinogenesis in Gastric Carcinoma. Chin J Clin Oncol 2002; 29: 709-712. Chinese.

74 Liu HJ, Gao H, Dong M, WanG L, Yuan Y: Pepsinogen C genetic polymorphism and gastric cancer susceptibility. Zhonghua Yi Xue Za Zhi 2002; 81: 947-948. Chinese.

75 Loktionov A: Common gene polymorphisms, cancer progression and prognosis. Cancer Lett 2004; 208: 1-33.

76 Zhou Y, Li N, Zhuang W et al: P53 codon 72 polymorphism and gastric cancer: a meta-analysis of the literature. Int J Cancer 2007; 121: $1481-1486$.

77 Pietsch EC, Humbey O, Murphy ME: Polymorphisms in the p53 pathway. Oncogene 2006; 25: 1602-1611.

78 Gemignani F, Moreno V, Landi S et al: A TP53 polymorphism is associated with increased risk of colorectal cancer and with reduced levels of TP53 mRNA. Oncogene 2004; 23: 1954-1956.
79 Kishi K, Kinoshita Y, Matsushima Y et al: Pepsinogen C gene product is a possible growth factor during gastric mucosal healing. Biochem Biophys Res Commun 1997; 238: 17-20.

80 Azuma T, Teramae N, Hayakumo $\mathrm{T}$ et al: Pepsinogen $\mathrm{C}$ gene polymorphisms associated with gastric body ulcer. Gut 1993; 34: $450-455$.

81 Brenner H, Arndt V, Stegmaier C et al: Is Helicobacter pylori infection a necessary condition for noncardia gastric cancer? Am J Epidemiol 2004; 159: 252-258.

82 Mitchell H, English DR, Elliott $\mathrm{F}$ et al: Immunoblotting using multiple antigens is essential to demonstrate the true risk of Helicobacter pylori infection for gastric cancer. Aliment Pharmacol Ther 2008; 28: 903-910.

83 Karnes Jr WE, Samloff IM, Siurala $M$ et al: Positive serum antibody and negative tissue staining for Helicobacter pylori in subjects with atrophic body gastritis. Gastroenterology 1991; 101: $167-174$.

Supplementary Information accompanies the paper on European Journal of Human Genetics website (http://www.nature.com/ejhg) 